\title{
Vitimização e Psicopatia em Autores de Violência Sexual contra Crianças e Adolescentes
}

\author{
Julia Nunes de Souza Teixeira ${ }^{a, b}$, Ana Cristina Resende $e^{a, 1}$, Rodrigo Perissinotto ${ }^{a, c}$ \\ a Pontifícia Universidade Católica de Goiás, Goiânia-GO, Brasil \\ ${ }^{\text {b }}$ Universidade do Estado do Rio de Janeiro, Rio de Janeiro-RJ, Brasil \\ c Centro Universitário de Mineiros, Mineiros-GO, Brasil
}

\section{RESUMO}

O objetivo do estudo foi investigar a vitimização em autores de violência sexual (AVS) contra crianças e adolescentes, assim como sua relação com traços de psicopatia. Participaram 30 reeducandos cumprindo pena em regime fechado por crimes sexuais contra crianças e adolescentes, que foram divididos em dois grupos: G1, AVS considerados psicopatas (PCL-R 230); G2, AVS não psicopatas (PCL-R<30). Os instrumentos utilizados no estudo foram: Psychopathy Checklist Revised (PCL-R) e Juvenile Victimization Questionnaire (JVQ). Para análise dos dados, foi utilizada estatística descritiva e comparativa por meio do Statistical Package for the Social Sciences (SPSS). Os resultados apontaram que participantes do G1 sofreram mais vitimizações durante a vida do que os participantes do G2.

Palavras-chave: autor de violência sexual; vitimização; psicopatia.

\section{ABSTRACT - Victimization and Psychopathy in Authors of Sexual Violence against Children and Adolescents}

The aim of the present study was to investigate psychopathy traits in offenders that committed sexual offences against children and adolescents, as well as their relationship with life experiences of victimization. The study consisted of 30 inmates serving prison sentences for sexual crimes against children and adolescents, who were divided into two groups: G1, composed of inmates considered psychopathic (PCL-R $\geq 30$ ); and G2, composed of inmates considered non-psychopathic (PCL-R $<30$ ). The results showed significant differences between the groups in relation to recidivism, escape and rebellion, showing that the psychopathic inmates were more undisciplined and more likely to reoffend than the non-psychopaths. In addition, there were indications that the G1 participants had suffered more victimization during their lives than those of the other group.

Keywords: Psychopathy; PCL-R; Sex Offender; Victimization.

\section{RESUMEN - Victimización y Psicopatía en Autores de Delitos Sexuales contra Niños y Adolescentes}

El objetivo del presente estudio fue investigar la victimización en agresores sexuales (AS) contra niños y adolescentes, así como su relación con rasgos de psicopatía. En el estudio participaron 30 reclusos cumpliendo condena en régimen cerrado por delitos sexuales contra niños y adolescentes, los reclusos fueron divididos en dos grupos: G1, AS considerados psicópatas (PCL-R $\geq 30$ ); G2, AS no psicópatas (PCL-R<30). Los instrumentos utilizados en el estudio fueron: Psychopathy Checklist Revised (PCL-R), y Juvenile Victimization Questionnaire (JVQ). Para el análisis de los datos se utilizó estadística descriptiva y comparativa mediante el Statistical Package for the Social Sciences (SPSS). Los resultados señalaron que los participantes del G1 sufrieron más victimizaciones durante su vida que los participantes del G2.

Palabras clave: agresión sexual; victimización; psicopatía.

No Brasil, o perfil mais comum do autor de violência sexual (AVS) contra crianças e adolescentes, encontrado em diferentes estudos, é um agressor homem, com idade entre 30 e 40 anos, em sua maioria de etnia parda, com ensino fundamental incompleto, solteiro e trabalhador braçal. Frequentemente, esse homem responde criminalmente apenas a processos sexuais, não possui histórico de reincidência criminal, é próximo de sua vítima (pai, padrasto, tio ou vizinhos, por exemplo), têm preferência por vítimas crianças e adolescentes e, geralmente, escolhe como local do abuso a residência da vítima ou a sua própria (Martins \& Jorge, 2010; Santos et al., 2015; Serafim, Saffi, Rigonatti, Casoy, \& Barros, 2009; Soares et al., 2016).

Várias características de personalidade são apontadas, em estudos realizados no Brasil, como presentes nos AVS. Dentre elas pode-se destacar a imaturidade geral, instabilidade afetiva e emocional, agressividade mediante à frustração, hostilidade com relação ao outro, autoestima rebaixada, insegurança e carência afetiva. Os AVS tendem a possuir sentimento de insegurança no que diz respeito a sua masculinidade, podendo apresentar forte 
componente sádico em sua personalidade, com grande potencial hostil, e muitas vezes evidenciando condutas semelhantes à personalidade antissocial (Costa \& Mello, 2012; Martins \& Jorge, 2010; Santos et al., 2015; Serafim et al., 2009; Soares et al., 2016).

A violência sexual contra crianças e adolescentes figura como uma das mais frequentes formas de violência à qual essa população é submetida (Brasil, 2013; Organização Mundial de Saúde [OMS], 2016). O impacto desse tipo de violência pode acompanhar a vítima e lhe trazer prejuízos que lhe seguirão ao longo da vida (Fergusson, McLeod, \& Horwood, 2013). Nesse sentido, faz-se necessário investimento em estratégias de prevenção para esse tipo de violência. No entanto, as estratégias de prevenção à violência sexual exigem a compreensão dos fatores que contribuem para que um indivíduo se torne um autor de violência sexual (AVS).

Estudos que buscam entender o que leva um homem a cometer um abuso sexual, mais especificamente contra crianças e adolescentes, que é o foco do presente artigo, apontam para múltiplos fatores causais. Dentre os fatores que os predispõem a estarem mais vulneráveis a cometer esse tipo de crime, os mais frequentemente especificados têm sido a presença de: parafilias, transtornos de personalidade, déficits cognitivos e alterações nos principais sistemas neuropsicológicos, fatores psicológicos, tais como problemas de empatia pelos outros, baixa autoestima e pouca capacidade de regular as emoções e impulsos. Ainda são apontados componentes biológicos (variações genéticas e neurobiológicas) que podem favorecer vulnerabilidades relacionadas a esse tipo de crime, além de fatores de nicho ecológico, ou seja, aprendizagem de circunstâncias sociais, econômicas, culturais e pessoais, como, por exemplo, crescer em família de baixo status socioeconômico e ser exposto a diferentes tipos de violência, como física, sexual ou psicológica (Barnett \& Mann, 2017; Gillespie \& Beech, 2017; Marshall, 2018; Ward \& Beech, 2016).

Segundo Ward e Beech (2016), a integridade dos aspectos psicológicos de uma pessoa advém da combinação de diferentes fatores, especialmente a herança biológica e o aprendizado social. Assim, os AVS adquirem vulnerabilidades psicológicas que os predispõem ao crime sexual. Os autores acrescentam que, além das vulnerabilidades psicológicas, o ambiente físico do indivíduo é importante contribuinte para a etiologia do crime sexual. Por exemplo, o meio pode disponibilizar possíveis vítimas e criar as circunstâncias específicas que desencadeiam os déficits psicológicos envolvidos. Nesse sentido, eventualmente, o fato de ter participado de uma guerra, ou o estresse do desemprego e ter que ficar em casa podem levar indivíduos a cometerem um crime sexual, o que, em outras circunstâncias, considerariam abominável. Em outras palavras, às vezes, os principais fatores causais que resultam em abusos sexuais residem no nicho ecológico, e não na pessoa.
Nessa mesma linha de raciocínio, pode-se considerar que o fato de ter sido vítima de abuso sexual ou físico na infância pode provocar desordens psicológicas, com risco acentuado para o desenvolvimento do abuso de álcool e drogas, bem como o funcionamento sexual adulto perturbado, pobre ajuste social, confusão sobre a identidade sexual, tentativas inapropriadas de reafirmar a masculinidade e recapitulação do abuso pela vítima em novas vítimas (Ward \& Beech, 2016).

No Canadá, há estudos (Greenberg, Firestone, Nunes, Bradford, \& Curry, 2005; Stirpe \& Stermac, 2003) apontando que os AVS contra crianças mostraram os maiores índices de vitimização sexual na infância ou antes dos 16 anos, e eram provenientes de famílias mais problemáticas do que os criminosos violentos e os criminosos não violentos pesquisados. Os dados indicaram também que a maior parte dos AVS reportou maior incidência de violência física na infância (maus-tratos), assim como dificuldades no ambiente familiar que era agressivo e desorganizado. Outro dado relevante advém do estudo de Stirpe e Stermac (2003), apontando que um terço dos indivíduos AVS por eles estudados reportou ter sido expulso de casa pelos pais antes dos 16 anos de idade.

Outra investigação científica realizada na França, Bélgica e Suíça, por Abbiati et al. (2014), corroborou as investigações anteriores que afirmam que os AVS, comumente, foram vítimas de violência física, psicológica ou sexual. Esse estudo apontou ainda que a vitimização por violência sexual foi mais frequente entre os AVS contra crianças e adolescentes, e que os pais, na maioria das vezes, eram os autores das agressões.

Assim, considerando a questão da vitimização de AVS, pesquisas realizadas em diferentes países apontam que experiências anteriores de vida e circunstâncias da vida atual, como, por exemplo, crescer com violência doméstica e exposição ao abuso sexual ou físico, juntamente com vida familiar conturbada e vivências de violência extrafamiliar, ofertavam grande potencial para suscitar o comportamento criminoso e de abuso sexual no futuro por parte desses indivíduos (Abbiati et al., 2014; Blackman \& Dring; 2016; Ferguson, Ogloff, \& Thomson, 2009; Forouzan \& Nicholls, 2015; Greenberg et al., 2005; Mendes \& França, 2012; Prentky, Knight, \& Lee, 2008; Stirpe \& Stermac, 2003).

Há que se destacar que também foram identificadas correlações positivas significantes entre ter sido vítima de abuso sexual na infância e traços de psicopatia no checklist de psicopatia (PCL-R). O diagnóstico de psicopatia em AVS pode ser feito por meio do PCL-R. Vários estudos têm demonstrado a eficiência dessa escala para tal diagnóstico. Harris, Rice e Lalumière (2001) investigaram relações de parentalidade antissocial com psicopatia e violência em 868 infratores. A parentalidade antissocial foi medida retrospectivamente usando indicadores como abuso infantil, negligência/rejeição, abuso em relação a outros membros da família e alcoolismo parental. Portanto, este estudo não 
examinou especificamente o abuso físico, mas sim uma ampla gama de variáveis relacionadas. No entanto, o abuso físico de crianças exibiu a carga mais forte na medida de pareamento antissocial, que foi significantemente relacionada $(r=0,32)$ aos sintomas psicopáticos. No entanto, neste estudo retrospectivo, não foi possível estabelecer se a paternidade antissocial influenciou as tendências psicopáticas de uma criança ou vice-versa.

O trabalho desenvolvido por Poythress, Skeem e Lillienfeld (2006) apontou que o abuso precoce pode estar diferencialmente relacionado aos dois fatores classicamente reconhecidos como componentes da psicopatia. Esses autores avaliaram sintomas psicopáticos, juntamente com ocorrências relatadas retrospectivamente de abuso físico, emocional e sexual precoce, em uma amostra de 702 infratores encarcerados da América do Norte. Os resultados indicaram significativa relação entre o grau geral de abuso precoce e os escores totais de psicopatia, mas essa relação foi atribuída principalmente aos escores no componente irresponsável - traços típicos de transtorno de personalidade antissocial (Fator 2), que normalmente é fruto de pessoas de se desenvolvem em contexto problemático ou família desestruturada. Nenhuma relação significativa foi evidente entre abuso na juventude e escores no componente interpessoal-afetivo (Fator 1), que são aqueles traços frequentemente observados em pessoas com psicopatia.

Gacon, Meloy e Bridges (2008), Hare (2006), Hill, Habermann, Klusmann, Berner e Briken (2008), Morana (2003) e Krstic et al.(2018) observaram que os AVS considerados psicopatas e antissociais tendiam a ser aqueles mais insensíveis afetivamente, com a capacidade empática muito diminuída, com comportamento impulsivo e desejosos de sensações estimulantes. Também se envolviam mais frequentemente com a criminalidade e abuso de substâncias psicoativas e possuíam conduta sexual marcada por crueldade, sendo que a vítima era reduzida a um mero objeto de degradação e rejeição.

Ao estudar a possibilidade de reincidência criminal, Mokros, Vohs e Habermeyer (2014) e Riquelme, Pérez e Muñoz (2004) avaliaram 60 AVS, evidenciando que AVS considerados psicopatas apresentavam maior probabilidade de reincidir criminalmente. Os resultados indicaram que esses AVS também apresentavam mais características interpessoais e emocionais próprias da psicopatia e tiveram pontuações significativamente maiores no PCL-R do que indivíduos condenados por outros tipos de crimes.

$\mathrm{Na}$ perspectiva de avaliar respostas a intervenção psicoterápica em AVS, Langton, Barbaree, Harkins e Peacock (2006) averiguaram a relação entre a reincidência criminal de AVS e os resultados apresentados por meio de tratamento cognitivo-comportamental. Identificaram correlação positiva entre altas pontuações do PCL-R e abandono do tratamento, apontando que os AVS com mais traços de psicopatia tenderam a interromper mais facilmente o tratamento.
Em um estudo mais recente, Krstic et al. (2018) utilizaram o PCL-R de quatro fatores com o objetivo de examinar traços psicopáticos em uma amostra com 958 AVS. Os fatores que avaliam a área afetiva e o comportamento antissocial foram preditores de crimes sexuais violentos. Os resultados mostraram que os AVS com maior número de traços de psicopatia prototípica (pontuação alta nos quatro fatores da escala e PCL-R total >30) cometeram crimes sexuais mais violentos do que os demais AVS.

Com base no exposto até o momento, depreende-se a relevância do exame do histórico de vida e características pessoais de AVS para compreensão de suas manifestações comportamentais, embasando eventuais propostas interventivas. Nessa perspectiva, o presente estudo teve como objetivo avaliar as vivências de vitimização entre adultos considerados psicopatas e não psicopatas em uma amostra de autores de violência sexual contra crianças e adolescentes.

\section{Método}

\section{Participantes}

Os participantes do estudo foram selecionados prática em uma penitenciária masculina da região CentroOeste do Brasil. Foram avaliados 30 reeducandos AVS contra crianças e adolescentes, o que correspondia a $22 \%$ de todos os condenados que cumpriam pena por crimes de natureza sexual contra crianças e adolescentes em regime fechado $(N=200)$ na penitenciária estudada. Eles foram selecionados aleatoriamente e as idades variaram de 18 a 60 anos. Os critérios de inclusão dos participantes da pesquisa foram: 1 . idade entre 18 e 60 anos; 2 . reeducandos do sexo masculino condenados por crimes sexuais; 3. cumprindo pena em regime fechado há pelo menos um ano; 4. ter vitimado crianças e/ou adolescentes. Os critérios de exclusão dos participantes foram: 1. reeducandos que não forneceram informações suficientes sobre seu histórico de vida durante a entrevista, sendo que, por esse critério, somente um dos voluntários foi excluído; 2. reeducandos cujos prontuários do cartório não ofertassem os dados necessários e relevantes à pesquisa.

Os 30 participantes foram divididos em dois grupos de estudo. O Grupo1 (G1) foi constituído por dez AVS com maior número de traços de psicopatia, ou seja, com pontuação maior ou igual a 30 no PCL-R que, de acordo com Young, Justice, \& Edberg (2010), DeMatteo et al. (2014) e Krstic et al. (2018), indicam traços suficientes para terem o diagnóstico de psicopatia nesse instrumento. O Grupo 2 (G2) foi constituído pelos demais $20 \mathrm{AVS}$, os quais tiveram pontuações menores do que 30 no PCL-R.

\section{Instrumentos}

Juvenile Victimization Questionnaire (JVQ). Trata-se de um questionário de autorrelato, composto por 33 itens breves e fechados, elaborados para reunir 
informações sobre uma ampla gama de vitimizações que abrangem cinco áreas gerais de preocupação (ou crivos): crime convencional (oito itens); maus tratos (quatro itens); vitimização por pares e irmãos (cinco itens); vitimização sexual (sete itens); vitimização indireta ou testemunho (nove itens) (Finkelhor, Hamby, Ormord, \& Turner, 2005). Nesse estudo, o instrumento foi baseado no JVQ traduzido e adaptado para adolescentes e adultos brasileiros (Faria, Zanini, Tolentino, Silva, \& Venâncio, 2017).

O crivo de crimes convencionais é composto por itens que investigam a vitimização por meio de roubo, assalto, vandalismo, agressão com ou sem arma de fogo, sequestro e preconceito. O crivo de maus-tratos abrange experiências vividas de abuso emocional, negligência e corresponde às vitimizações que têm, como agressor, pessoas da família e cuidadores. O crivo das vitimizações por pares aborda as agressões efetuadas por pessoas de idade semelhante ou irmãos, como bullying, ataques aos genitais sem cunho sexual e violência por namorado(a). O crivo das vitimizações sexuais engloba agressão sexual por adulto, por pares e assédio sexual. O crivo da vitimização indireta aborda o testemunhar de violência sofrida por outras pessoas, como testemunho de violência doméstica, assassinato, terrorismo ou motins e exposição a conflitos étnicos.

O questionário avalia as vitimizações sofridas tanto ao longo da vida, como no último ano. No presente estudo, foram utilizadas apenas as respostas ao longo da vida, desconsiderando o período em que se encontravam presos, pois o objetivo era verificar se existia relação entre a vitimização sofrida e o crime cometido.

Psychopathy Checklist-Revised (PCL-R). Escala desenvolvida e validada por Rorbert Hare (Hare, 1991 e 2003) nos Estados Unidos para avaliar a psicopatia em populações forenses masculinas. No Brasil, foi validada por Morana (2004), que apresentou índice de confiabilidade interavaliadores ICC (intraclass correlation coefficient), para uma amostra de prisioneiros, igual a 0,91 . Neste estudo, foi utilizado o ponto de corte de 30 pontos para os participantes que compuseram o G1, que foi o ponto de corte estabelecido por Hare (1991) para identificar indivíduos com maior número de traços de psicopatia. Esse ponto de corte também foi utilizado por Young et al. (2010), DeMatteo et al. (2014) e Krstic et al. (2018) para discriminar os participantes que preenchiam critérios para serem considerados psicopatas ( $\geq 30$ pontos). Os demais participantes, com pontuação abaixo de 30 , constituíram o G2.

\section{Procedimentos}

Primeiramente, o projeto de pesquisa foi aprovado pela direção da penitenciária que aceitou participar do estudo, sendo a seguir analisado e aprovado por um Comitê de Ética em Pesquisa (CAAE 0110.0.168.168-11). Em seguida, foi feita a coleta de dados nos prontuários no cartório da penitenciária de AVS contra crianças e adolescentes, que foram escolhidos aleatoriamente. Aqueles reeducandos que aceitaram participar do estudo assinaram o Termo de Consentimento Livre e Esclarecido (TCLE) e, logo em seguida, foi realizada a entrevista, sendo convidados os indivíduos até se completar 30 casos na amostra.

O tempo para coletar os dados de cada participante foi de aproximadamente três horas e trinta minutos. Foi necessária uma hora para levantar as informações no processo do cartório (análise documental), duas para a entrevista do PCL-R e, aproximadamente, quinze minutos para o JVQ.

Posteriormente, $60 \%$ dos protocolos do PCL-R $(N=20)$ foram classificados aleatoriamente e corrigidos por dois juízes, para o cálculo da análise de concordância entre os avaliadores, por meio do cálculo do Coeficiente de Correlação Intraclasses (ICC). O ICC médio foi de 0,89 (variando entre 0,62 e 0,96 ) e a mediana atingiu 0,91. Esses valores podem ser considerados bons (entre 0,60 e 0,74) e excelentes (acima de 0,74) (Cicchetti, 1994; Hunsley \& Mash, 2008; Shrout \& Fleiss, 1979). Outra análise de fidedignidade foi realizada por meio do coeficiente alfa de Cronbach. Para o Fator 1 (traços de psicopatia), o coeficiente alfa foi 0,79 e para o F2 (traços antissociais) foi 0,94 . No geral, a consistência interna do total de pontuações com o PCL-R pode ser considerada aceitável para uma escala clínica, com índice de 0,93 para todos os itens.

Após as análises de fidedignidade do PCL-R, seguiu-se com os levantamentos de pontos alcançados nos dois instrumentos utilizados e as análises foram realizadas por meio de estatísticas descritivas e comparativas utilizando o Statistical Package for the Social Sciences (SPSS), versão 20.0. As variáveis qualitativas foram apresentadas como frequência absoluta e relativa e as quantitativas como média e desvio padrão (DP). Verificou-se a diferença estatística entre os grupos de participantes (G1 versus G2) por meio do teste de qui-quadrado ou Exato de Fisher para as variáveis qualitativas e, para as quantitativas, o teste $t$ de Student para amostras independentes. O teste $t$ de Student foi utilizado, mesmo na ausência de normalidade da maioria das variáveis, utilizando a técnica de reamostragem de bootstrap, método que ajusta a normalidade das variáveis em amostras pequenas, aproximando a média amostral ou diferença entre as médias a uma distribuição normal (Curran-Everett et al., 2017).

Além disso, foram verificados os tamanhos dos efeitos das diferenças entre os grupos e algumas variáveis utilizando o $d$ de Cohen para as variáveis quantitativas $\mathrm{e}$ o $V$ de Cramer para as qualitativas. O tamanho do efeito foi classificado como pequeno $(d=0,20$ a 0,49$)$, médio $(d=0,50$ a 0,79$)$ e grande $(d>0,80)$ com base nos valores de referência sugeridos por Cohen (1988). Para o v de Cramer, o efeito foi classificado como pequeno $(v=0,10$ a 0,20$)$, médio $(v=0,21$ a 0,60$)$ e grande $(v=0,61$ a 1,0$)$, usando como referência os valores sugeridos por Rea e Parker (1992). 
Também foi verificada a relação entre variáveis sociodemográficas, perfil criminal e vitimizações mensurados pelo JVQ e as pontuações do PCL-R utilizando o coeficiente de correlação de Pearson. Em todas as análises os valores de $p<0,05$ foram considerados estatisticamente significativos.

\section{Resultados e Discussão}

\section{Dados Sociodemográficos e Criminais}

Quanto ao perfil sociodemográfico dos participantes, a idade variou entre 18 e 60 anos, com uma média de 33,5 anos $(D P=9,2)$, a escolaridade da maioria $(63,3 \%$, $n=19)$ era de cinco a oito anos de estudo e a raça/cor da pele autodeclarada foi de 43,3\% $(n=13)$ de brancos, seguidos de 30\% (n=9) de raça parda e 13,3\% $(n=4)$ da raça negra. Com relação ao estado marital, $40 \%(n=12)$ eram casados. Do total de participantes, $46,7 \%(n=14)$ possuíam filhos, sendo a média de filhos de 2,9 $(D P=2,2$; Mínimo: 1, Máximo: 9). A profissão predominante foi o trabalho braçal, especialmente da construção civil com $40,0 \% \quad(n=12)$, seguido de manutenção/serviços gerais com $16,7 \%(n=5)$, vigilante/motorista com $13,3 \%$ $(n=4)$, autônomo com $10 \%(n=3)$ e trabalhador rural também com $10 \%(n=3)$ dos participantes.

Tomando como base os dados coletados e analisados, o perfil mais frequente de AVS contra crianças de adolescentes do sexo masculino neste estudo foi: idade entre 30 e 40 anos, em sua maioria de raça/cor de pele branca, com ensino fundamental incompleto, solteiro e trabalhador braçal (predominantemente da construção civil). Esses achados confirmam o perfil encontrado para AVS contra crianças e adolescentes em outros estudos realizados no Brasil (Huss, 2011; Martins \& Jorge, 2010; Santos et al., 2015; Serafim et al. 2009; Soares et al., 2016).

No que tange ao perfil criminal, a idade média das vítimas foi de 10,0 anos $(D P=2,9$; mínimo: 5 ; máximo: $15), 66,7 \%(n=20)$ tinha algum tipo de proximidade do agressor (pai, padrasto ou tio), sendo a maioria deles $(85 \%, n=17)$ pais ou padrastos. O local do crime em $50 \%$ $(n=15)$ das vezes foi na residência da vítima, em $26,7 \%$ $(n=8)$ foi na residência do AVS, em $10 \%(n=3)$ foi em um terreno baldio ou matagal e, em 13,3\%, $(n=4)$ ocorreu em outro local.

Ao se tomar os dados sociodemográficos e criminais por grupo, observou-se que G1 apresentou média de idade (G1: $M=28,4, D P=7,6 ; \mathrm{G} 2: M=36,1, D P=9,2)$ significativamente menor $(p=0,029)$ e médias maiores do ponto de vista estatístico nas variáveis: número de vítimas ( $p=0,006$; G1: $M=2,6$ e G2=1,3), de fugas $(p=0,009 ; \mathrm{G} 1=2$ e $\mathrm{G} 2=0,4)$, de participação em rebeliões $(p=0,014, \mathrm{G} 1=0,6$ e $\mathrm{G} 2=0,1)$, de tempo total de pena ( $p=0,001$; $\mathrm{G} 1=34,1$ anos e $\mathrm{G} 2=14,2)$, vítima adolescente e adulta $(p=0,024$; G1=1,1 e G2=0,1). Em todas essas variáveis o tamanho do efeito ( $d$ de Cohen) foi grande, variando de 0,70 a 1,21. O G1 também responde por maior variedade de crimes em relação ao G2 $(p<0,05$; G1 apresentou em média cinco tipos de crimes diferentes por pessoa, enquanto do G2 tem em média dois). Por sua vez, G2 evidenciou mais proximidade (parentesco) com a vítima (85\% do G2) que o G1 (30\% dos AVS tinham parentesco com suas vítimas), e é mais propenso a escolher a própria residência como local do abuso (40\% dos AVS do G2 e nenhum caso em G1). O tamanho do efeito mensurado pelo $v$ de Cramer mostrou os seguintes efeitos: associação relativamente forte entre os grupos e versatilidade criminal $(v$ Cramer $=0,446)$, proximidade com a vítima $(v$ Cramer $=0,585)$ e local do crime $(v$ Cramer $=0,536$ ).

Os dados apontam que um terço dos AVS contra crianças e adolescentes $(N=10 ; 33,3 \%)$ preenchiam os critérios para o diagnóstico de psicopatia (conforme o critério de pontuação maior ou igual a 30 no PCL-R). A incidência de psicopatia em AVS, encontrada neste estudo, é ligeiramente maior que aquela encontrada em um estudo realizado por Young et al. (2010), em que a incidência foi de $28 \%$ dos AVS (PCL-R>30).

Diferenças significativas foram observadas entre os grupos, G1 (considerados psicopatas) e G2 (considerados não psicopatas), com relação a algumas variáveis coletadas por meio dos processos que estavam disponíveis no cartório da penitenciária. O grupo de participantes considerados psicopatas prejudicou um número significativamente maior de vítimas do que os participantes considerados não psicopatas. Além disso, há significativamente mais registros de fugas, rebeliões, versatilidade criminal, maior o número de processos de modo geral e de processos por crimes sexuais, o que, consequentemente, faz com que o tempo total de pena seja também significativamente maior no G1.

Ainda com relação ao G1, esse grupo possuía significativamente mais traços de psicopatia (componente interpessoal-afetivo = fator F1), corroborando evidências da literatura científica sobre o tema (Krstic et al., 2018; Olver \& Wong, 2009; 2015; Young et al., 2010), como também significativamente mais traços de transtorno de personalidade antissocial TPA (fator F2) e, consequentemente, maior pontuação no PCL-R. O presente estudo ratifica os achados de Krstic et al. (2018), em que os autores de violência sexual com características prototípicas de psicopatia evidenciaram pontuações maiores nos fatores do PCL-R e mais quantidade de crimes sexuais. Tais observações em relação ao G1 indicam que esse grupo foi composto por indivíduos mais indisciplinados no sistema carcerário e com mais chances de reincidirem criminalmente (Balsis, Busch, Wilfong, Newman, \& Edens, 2017; Gacono, Meloy, \& Bridges, 2008).

\section{Vitimizações Sofridas ao Longo da Vida}

Sobre as vitimizações sofridas, considerando o grupo total avaliado, a mais frequente foi a vitimização testemunhada e indireta, depois o crime convencional, os 
maus tratos, a vitimização por pares ou irmãos e, por último, a vitimização sexual. $\mathrm{Na}$ vitimização testemunhada e indireta, todos os AVS $(N=30,100,0 \%)$ foram revitimizados nesse crivo, ou seja, sofreram entre três a sete tipos de vitimização testemunhada e indireta, sendo as mais citadas: ver alguém ser atacado sem uso de objetos (item selecionado por $100 \%$ dos participantes); ver alguém ser atacado com uso de objetos $(86,7 \%, n=26)$; ouvir tiros, bombas ou motins na rua $(63,3 \%, n=19)$.

No crivo de crime convencional, apenas um AVS $(3,3 \%)$ relatou que não sofreu esse tipo de vitimização. Dez por cento $(n=3)$ dos AVS relaram que sofreram um único tipo de vitimização neste crivo, e $86,7 \%(n=26)$ dos AVS foram revitimizados por meio de três a oito tipos de vitimização, considerando que este crivo contém oito itens. Nessa categoria de vitimização, as mais frequentemente citadas foram: ser atacado de propósito com objetos ou armas $(86,7 \%, n=25)$; ser atacado sem uso de objetos ou armas $(66,7 \%, n=20)$; ser roubado $(63,3 \%, n=19)$.

Com relação à vitimização sofrida no crivo de maus-tratos, $6,7 \%(n=2)$ dos participantes relataram que não sofreram nenhuma vitimização deste tipo, 16,7\% $(n=5)$ relataram que sofreram esse tipo de violência uma vez e $76,6 \%(n=23)$ foram revitimizados por meio de dois a quatro tipos de vitimização (considerando as quatro possibilidades desse crivo). Os maus tratos mais comuns foram: ter medo ou se sentir muito mal porque alguém o "xingou" ou chamou de outros nomes ou disse coisas ofensivas para a pessoa ou disseram que não o queria mais (item citado por $66,7 \%, n=20$ ); apanhar ou ser machucado fisicamente de alguma forma $(76,6 \%, n=23)$; um familiar evitar o contato do participante com um membro da família ou alguém importante para ele $(43,3 \%, n=13)$.

$\mathrm{Na}$ vitimização por pares ou irmãos, $6,7 \%(\mathrm{n}=2)$ dos participantes declararam que não sofreram este tipo de vitimização, $16,7 \%(n=5)$ declararam que sofreram esse tipo de violência uma vez e 76,6\% $(n=23)$ foram revitimizados. Esses participantes revitimizados sofreram entre dois e cinco tipos de vitimização por pares ou irmãos, sendo as vitimizações por pares mais frequentemente citadas: apanhar de alguém $(50,0 \%, n=15)$, ser azucrinado ou perseguido para fazer algo que não queria fazer $(43,3 \%, n=13)$, ser atacado por um grupo ou gangue $(33,3 \%, n=10)$.

No que tange à vitimização sexual, $20,0 \%(n=6)$ dos AVS declararam que nunca sofreram nenhum tipo de vitimização sexual; 33,3\% $(n=10)$ dos AVS declararam que sofreram esse tipo de violência uma vez e 46,7\% $(n=14)$ foram revitimizados nesse crivo. Esse tipo de vitimização normalmente ocorreu nas seguintes circunstâncias: ser forçado a fazer sexo sem querer $(40 \%, n=12)$ ou fazer algo relacionado a sexo $(33,3 \%, n=10)$.

Considerando esse levantamento geral das porcentagens dos cinco diferentes tipos de vitimizações sofridas pelos AVS ao longo da vida, por meio do autorrelado no
JVQ, observou-se que os índices foram maiores quando comparados com um levantamento realizado por Campos (2016) com amostra de universitários de idade semelhante (18 a 56 anos) ao presente estudo (18 a 60 anos), da mesma região do Brasil em que foi conduzido. Campos (2016) considerou a prevalência de vitimização e revitimização no JVQ muito altas nos universitários, em que as menores porcentagens foram de $71,0 \%$ e $38,3 \%$ para a vitimização e revitimização sexual respectivamente, e $95,0 \%$ e $80,9 \%$ para vitimização e revitimização por violência indireta ou testemunhada. Entre os AVS examinados nesse trabalho, os índices foram ainda maiores, ou seja, $80,0 \%$ e $46,7 \%$ para vitimização e revitimização sexual, e 100,0\% para vitimização revitimização por violência indireta ou testemunhada.

Nos dois estudos, os índices de vitimização e revitimização testemunhada foram os mais expressivos, independente do contexto observado, universitário ou presidiário. Destaca-se que a exposição a uma vizinhança violenta ou a exposição indireta à violência, como ser próximo de uma pessoa que seja autora ou vítima de atos violentos, tende a ser referido como um dos principais componentes para o surgimento de problemas de comportamento.

Entende-se que as vitimizações em geral e, especialmente a sexual, do grupo de AVS, quando integradas aos vários outros fatores apontados na literatura científica (transtornos de personalidade; déficits cognitivos e alterações nos principais sistemas neuropsicológicos; fatores psicológicos, genéticos e fatores de nicho ambiental) poderiam favorecer vulnerabilidades relacionadas ao cometimento de crimes sexuais (Greenberg et al. 2005; Stirpe \& Stermac, 2003; Ward \& Beech, 2016).

De qualquer forma, a vitimização sexual dos AVS reforça a ideia de que a maioria dos AVS também foram vítimas desse tipo de crime. Pesquisas realizadas por Stirpe e Stermac (2003) e Greenberg et al. (2005) no Canadá e por Abbiati et al. (2014) na França, Bélgica e Suíça fortalecem esses achados. Os resultados dessas três investigações apontaram que os AVS contra crianças mostram histórico de maior índice de vitimização sexual na infância. Greenberg et al. (2005) e Abbiati et al. (2014) acrescentam que os AVS também foram mais vitimizados física e psicologicamente, o que também corrobora os resultados do presente estudo.

Ao retomar a comparação dos atuais achados com os encontrados por Campos (2016), nota-se maior discrepância no crivo da vitimização por pares. No presente estudo foi observada porcentagem de revitimização em 76,6\% dos participantes, enquanto Campos (2016) identificou índice de $46,7 \%$. Ou seja, os AVS relataram frequência mais elevada de revitimização, atingindo $29,9 \%$ a mais do que os universitários. Nos demais crivos, de vitimização convencional e maus tratos, os AVS relataram $17,1 \%$ e $22,5 \%$ mais revitimização que os universitários. 
A vitimização no crivo de maus-tratos foi muito frequente no presente estudo, sendo que 93,3\% dos AVS referiram-se vítimas desse tipo de violência. Embora o fato de terem sido vitimizados não justifique o crime cometido, a vitimização pode influenciar na prática de crimes. Ferguson et al. (2009) reforçam essa afirmação ao apontarem que maus-tratos na infância estão diretamente relacionados à prática de crimes na adolescência e na vida adulta. Blackman e Dring (2016) ratificam esses dados ao afirmarem que grande parte dos AVS sofreu algum tipo de negligência ou maus-tratos durante a infância.

Esses dados sobre vitimização e revitimização também concordam com o que Marshall (2018) e Ward e Beech (2016) sustentam sobre a questão dos fatores de nicho ecológico em AVS. Ou seja, a aprendizagem de circunstâncias sociais, econômicas, culturais e pessoais, como, por exemplo, crescer em família de baixo status socioeconômico (como no caso dos AVS que possuíam, em sua maioria, ensino fundamental incompleto e falta de qualificação profissional), e ser exposto a diferentes tipos de violência, como física, sexual ou psicológica, podem ser fatores que predispõem o AVS a cometer crimes sexuais, embora a vitimização por si só não seja suficiente para explicar a conduta violenta dessas pessoas. Contudo, à medida que a violência cresce, os indivíduos tornam-se mais suscetíveis a se tornarem vítimas desse fenômeno, assim como à repetição dele, passando, portanto, a ser um fenômeno social, e não mais individual, como afirma Waiselfisz (2014, 2015).

Ao comparar os dois grupos, considerando a pontuação total no JVQ, G1 relatou significativamente mais experiência de vitimização que G2 $(p=0,003)$ ao longo da vida, com exceção do crivo de vitimização convencional. A vitimização convencional pode ser considerada igualmente alta nos dois grupos. O tamanho do efeito para todas essas experiências foi grande, variando entre 0,82 e 0,90, com exceção do tamanho do efeito para o crivo de crimes convencionais (ver Tabela 1).

Tabela 1

Comparação Estatística entre os Grupos com Relação às Vitimizações Sofridas no ao Logo da Vida e em cada Crivo do JVQ

\begin{tabular}{|c|c|c|c|c|c|c|c|c|c|}
\hline & Vitimização & Grupo & $\mathrm{M}$ & DP & Min & $\operatorname{Max}$ & $t$ & $p$ & $d$ \\
\hline & & G1 & 21,9 & 5,5 & 15 & 31 & 321 & 0003 & 1274 \\
\hline Vitin & nização ao longo da vida & G2 & 14,5 & 6,1 & 3 & 26 & 3,21 & 0,003 & 1,214 \\
\hline & & GT & 17,0 & 6,8 & 3 & 31 & & & \\
\hline & & G1 & 5,6 & 2,5 & 2 & 12 & & & \\
\hline & Convencional & G2 & 4,1 & 2,5 & 0 & 9 & כבנוג & 0,132 & 0,000 \\
\hline & & GT & 4,6 & 2,5 & 0 & 12 & & & \\
\hline & & G1 & 3,8 & 0,8 & 3 & 5 & 01 & 0050 & 0 \\
\hline & Maus Tratos & G2 & 2,7 & 1,6 & 0 & 5 & 2,01 & 0,000 & 0,00 \\
\hline$\bigcup_{\pi}^{n}$ & & GT & 3,1 & 1,5 & 0 & 5 & & & \\
\hline$\ddot{\rightleftarrows}$ & & G1 & 4,3 & 1,6 & 1 & 6 & 256 & 0021 & 1161 \\
\hline 趸 & Por Pares & G2 & 2,8 & 1,5 & 0 & 6 & ט, & $0,0<1$ & 1,101 \\
\hline ه & & GT & 3,3 & 1,6 & 0 & 6 & & & \\
\hline . & & G1 & 2,3 & 1,4 & 0 & 5 & ? & 0010 & 1020 \\
\hline & Sexual & G2 & 1,1 & 1,2 & 0 & 5 & ה & $0,0=0$ & ט, \\
\hline & & GT & 1,6 & 1,3 & 0 & 5 & & & \\
\hline & & G1 & 8,3 & 2,0 & 6 & 12 & 12 & 0018 & 0021 \\
\hline & Indireto & G2 & 6,3 & 2,8 & 2 & 12 & 2,12 & & \\
\hline & & GT & 7,0 & 2,7 & 2 & 12 & & & \\
\hline
\end{tabular}

Nota. G1=indivíduos com PCL-R $\geq 30$ (N=10); G2=indivíduos com PCL-R<30 (N=20); G1+G2=GT, total da amostra (N=30)

$\mathrm{Na}$ década de 1990, diferentes estudos apontaram a relação entre os tipos vitimização (abuso sexual, juntamente com o abuso físico e negligência de crianças) e prisão de adultos por crimes sexuais e traços de psicopatia (Luntz \& Widom, 1994; Weiler e Widom, 1996; Widom \& Ames, 1994). Posteriormente, Poythress et al.
(2006) também identificaram que o abuso sexual, emocional ou físico precoce poderiam estar relacionados à psicopatia. A partir dos dados coletados, notou-se que os AVS psicopatas, além de serem mais indisciplinados no sistema prisional, apresentando maior número de fugas, rebeliões, maior versatilidade criminal e maior 
tempo total de pena, também foram mais vitimizados que os demais AVS, acompanhando achados da literatura científica da área.

Vale lembrar que como o JVQ é um instrumento de autorrelato, algumas respostas podem corresponder a simulação (faking). Os AVS podem ter dado respostas que os colocassem como vítimas, como pessoas que sofreram violências ao longo da vida. Vitimização indireta e maus tratos também foram mais frequentes no G1 (psicopata). De modo semelhante ao que pode ter ocorrido no grupo todo, esse dado pode ter sofrido simulação, onde os AVS com mais traços de psicopatia podem ter oferecido respostas que os colocassem como vítimas da sociedade. Porém, as associações empíricas feitas no presente estudo falseiam essas suspeitas. Por conseguinte, este estudo revelou que os AVS psicopatas relataram significantemente mais experiência de maus tratos, vitimizações por pares, testemunhada ou sexual durante a vida, o que mostra relevante e positiva associação entre estas vitimizações e psicopatia nessa amostra de AVS.

\section{Referências}

Abiatti, M., Mezzo, B., Waeny-Desponds. J., Minervini, J., Mormont, C., \& Gravier, B. (2014). Victimization in childhood of male sex offenders: Relationship between violence experienced and subsequent offenses through discourse analysis. Victims and Offenders, 9(2), 234-254. doi:10.1080/15564886.2014.881763

Balsis, S., Busch, A. J., Wilfong, K. M., Newman, J. W., \& Edens. J. F. (2017): A statistical consideration regarding the threshold of the psychopathy checklist - revised. Journal of Personality Assessment, 99(5), 494-502. doi:10.1080/00223891.2017.1281819

Barnett G. D., \& Mann R. E. (2017). Theories of empathy deficits in sexual offenders. Em D. P. Boer (Eds). The Wiley handbook on the theories, assessment, and treatment of sexual offending (pp. 223-244), Vol 1, Theories. Chichester: John Wiley \& Sons.

Blackman, J. S., \& Dring, K. (2016). Sexual agression against children: Pedophiles' and Abusers' Development, Dynamics, Treatability, and the Law. Routledge, New York.

Brasil, Ministério da Saúde, Secretaria de Vigilância em Saúde, Departamento de Vigilância de Doenças e Agravos Não Transmissíveis e Promoção da Saúde. (2017). Viva: Vigilância de Violências e Acidentes: 2013 e 2014. Brasília: Ministério da Saúde. Recuperado de http:// bvsms.saude.gov.br/bvs/publicacoes/viva_2013_2014.pdf

Campos, D. C. (2016). Vitimização e revitimização em estudantes universitários: Influência de fatores individuais e sociais (Tese de doutorado não publicada). Pontifícia Universidade Católica de Goiás, Goiânia. Recuperado de http://tede2.pucgoias.edu.br:8080/handle/tede/1773

Cicchetti, D. V. (1994). Guidelines, criteria, and rules of thumb for evaluating normed and standardized assessment instruments in psychology. Psychological Assessment, 6(4), 284-290. doi: 10.1037/1040-3590.6.4.284

Costa, C. S., \& Mello, M. F. (2012). Indicadores comportamentais de propensão ao homicídio em agressores sexuais. Jornal Brasileiro de Psiquiatria, 61(1), 33-38. doi:10.1590/S0047-20852012000100007

Curran-Everett, D. (2017). Explorations in statistics: The assumption of normality. Advances in Physiology Education, $41(3), 449-453$. doi:10.1152/advan.00064.2017

Faria, M. R. G., Zanini, D. S., Tolentino, G. P., Silva, I. O., \& Venâncio, P. E. M. (2017). Tradução e dados preliminares do instrumento de vitimização para adolescentes e adultos brasileiros a partir do Juvenile Victimization Questionnaire. Revista Portuguesa de Ciências do Desporto, 17(S3.A), 25-36. Recuperado de https://rpcd.fade.up.pt/_arquivo/artigos_soltos/2017-S3A/02.pdf

DeMatteo, D., Edens, J. F., Galloway, M., Cox, J., Smith, S. T., Koller, J. P., \& Bersoff, B. (2014). Investigating the role of the Psychopathy Checklist - Revised in United States case law. Psychology, Public Policy and Law, 20(1), 96-107. doi:10.1037/a0035452

Fergusson, D. M., McLeod, G. F. H., \& Horwood, L. J. (2013). Childhood sexual abuse and adult developmental outcomes: Findings from a 30-year longitudinal study in New Zealand. Child Abuse and Neglect, 37(9), 664-674. doi:10.1016/j.chiabu.2013.03.013

Ferguson, A. M., Ogloff, J. R. P., \& Thomson, L. (2009). Predicting recidivism by mentally disordered offenders using the LSI-R:SV. Criminal Justice and Behavior, 3(1), 5-20. doi:10.1177/0093854808326525

Finkelhor, D., Hamby, S. L., Ormrod, R., \& Turner, H. (2005). The juvenile victimization questionnaire: Reliability, validity, and national norms. Child Abuse \& Neglect, 29(4), 383-412. doi:10.1016/j.chiabu.2014.11.001

Forouzan, E., \& Nicholls, T. L. (2015). Childhood and adolescent characteristics of women with high versus low psychopathy scores: Examining developmental precursors to the malignant personality disorder. Journal of Criminal Justice, 43(4), 307-320. https://doi. org/10.1016/j.jcrimjus.2015.06.001

Gacono, V. B., Meloy, J. R., \& Bridges, M. (2008). A Rorschach understanding of psychopats, sexual homicide perpetrators, and nonviolent pedophiles. Em C. Gacono \& B. Evans (Eds). The Handbook of Forensic Rorschach Assessment (pg. 361-378). New York, Routledge.

Gillespie, S. M., \& Beech, A. R. (2017) Theories of emotion regulation. Em D. P. Boer. (Ed). The Wiley handbook on the theories, assessment, and treatment of sexual offenders (pp. 245-263), Vol 1, Theories. Chichester: John Wiley \& Sons.

Greenberg, D. M., Firestone, P., Nunes, K. L., Bradford, J. M., \& Curry, S. (2005). Biological fathers and stepfathers who molest their daughters: Psychological, phallometric, and criminal features. Sexual Abuse: A Journal of Research and Treatment, 17(1), 39-46. doi: $10.1177 / 107906320501700105$

Hare, R. D. (1991). The hare Psychopathy Checklist-Revised (PCL-R). Toronto: Multi-Health Systems.

Hare, R. D. (2003). Hare Psychopathy Checklist - Revised technical manual (2nd ed.). Toronto: Multi-Health Systems.

Hare, R. D. (2006). Psychopathy: A clinical and forensic overview. Psychiatric Clinic of North American, 29(3), 709-724. doi:10.1016/j. psc.2006.04.007

Harris, G. T., Rice, M. E., \& Lalumière, M. (2001). Criminal Violence: The Roles of Psychopathy, Neurodevelopmental Insults, and Antisocial Parenting. Criminal Justice and Behavior, 28(4), 402-426. https://doi.org/10.1177/009385480102800402

Hill, A., Habermann, N., Klusmann, D., Berner, W., \& Briken, P. (2008). Criminal recidivism in sexual homicide perpetrators. International Journal of Offender Therapy and Comparative Criminology, 52(1), 5-20. doi:10.1177/0306624x07307450 
Hunsley, J., \& Mash, E. J. (2008). A Guide to Assessments that Work. New York: Oxford University Press.

Huss, M. T. (2011). Psicologia forense: Pesquisa, prática clínica e aplicações. Porto Alegre: Artmed.

Krstic, S., Neumann, C. S., Roy, S., Robertson, C.A., Knight, R. A., \& Hare, R. D. (2018). Using Latent Variable- and Person-Centered Approaches to Examine the Role of Psychopathic Traits in Sex Offenders. Personality Disorders, 9(3), 207-216. doi: 10.1037/per0000249

Langton, C. M., Barbaree, H. E., Harkins, L., \& Peacock, E. J. (2006). Sex offenders' response to treatment and its association with recidivism as a function of psychopathy. Sexual Abuse: A Journal of Research and Treatment, 18(1), 99-120. doi: 10.1177/107906320601800107

Luntz, B. K., \& Widom, C. S. (1994). Antisocial personality disorder in abused and neglected children. American Journal of Psychiatry, 151(5), 670-674. doi: 10.1176/ajp.151.5.670

Marshall, W. L. (2018). A brief history of psychological theory, research, and treatment with adult male sex offenders. Current psychiatry reports, 20(8), 57. doi: 10.1007/s11920-018-0920-0

Martins, C. B. G., \& Jorge, M. H. P. M. (2010). Abuso sexual na infância e adolescência: Perfil das vítimas e agressores em município do sul do Brasil. Texto Contexto Enfermagem, 19(2), 246-255. doi:10.1590/S0104-07072010000200005

Mendes, A. P. N., \& França, C. P. (2012). Contribuições de Sándor Ferenczi para a compreensão dos efeitos psíquicos da violência sexual. Psicologia em Estudo, 17(1), 121-130. doi: 10.1590/s1413-73722012000100014

Mokros, A., Vohs, K., \& Habermeyer, E. (2014). Psychopathy and violent reoffending in German-speaking countries. European Journal of Psychological Assessment, 30(2), 117-129. doi:10.1028/1015-5759

Morana, H. C. P. (2003). Identificação do ponto de corte para a escala PCL-R (Psychopathy Checklist Revised) em população forense brasileira: Caracterização de dois subtipos de personalidade; transtorno global e parcial (Tese de doutorado não publicada). Faculdade de Medicina da Universidade de São Paulo, São Paulo. Recuperado de https://www.teses.usp.br/teses/disponiveis/5/5142/tde-14022004-211709/pt-br.php

Olver, M. E., \& Wong, S. C. P. (2009). Therapeutic responses of psychopathic sexual offenders: Treatment attrition, therapeutic change, and long-term recidivism. Journal of Consulting and Clinical Psychology, 77(2), 328-336. doi: 10.1037/a0015001

Olver, M. E., \& Wong, S. C. P. (2015). Short- and long-term recidivism prediction of the PCL-R and the effects of age: A 24-year follow-up. Personality Disorders: Theory, Research, and Treatment, 6(1), 97-105. doi: 10.1037/per0000095

Organização Mundial de Saúde [OMS]. (2016). Maltrato infantil. Nota descritiva. Recuperado de http://www.who.int/mediacentre/factsheets/ fs150/es/

Poythress, N. G., Skeem, J. L., \& Lilienfeld, S. O. (2006). Associations among early abuse, dissociation, and psychopathy in an offender sample. Journal of Abnormal Psychology, 115(2), 288-297. doi: 10.1037/0021-843X.115.2.288

Rea, L. M., \& Parker, R. A. (1992). Designing and conducting survey research. San Francisco: Jossey-Bass.

Prentky, R. A., Knight, R. A., \& Lee, A. F. S. (2008). Child sexual molestation. Em C. R. Bartol \& A. M. Bartol. Current Perspectives in Forensic Psychology and Criminal Behavior (pp. 123-134) ed. 2. Los Angeles: Sage.

Riquelme, C. A., Pérez, N., \& Muñoz, C. G. (2004). Adaptación de la Escala de Calificación de la Psicopatía Revisada (PCL-R) de Robert Hare en población reclusa del Centro de Detención Preventiva de San Miguel (Monografia não publicada). Facultad de Ciencias Sociales, Universidade do Chile, Chile. Recuperado de http://www.tesis.uchile.cl/tesis/uchile/2004/abalos_c/sources/abalos c.pdf

Santos, C. A., Costa, M. C. O., Amaral, M. T. R., Nascimento Sobrinho, C. L., Musse, J. O., \& Costa, A. M. (2015). Agressor sexual de crianças e adolescentes: Análise de situações relacionadas à violação e vítimas. Adolescência e Saúde, 12(3), 7-20. Recuperado de http:// www.adolescenciaesaude.com/detalhe_artigo.asp?id=519

Serafim, A. D. P., Saffi, F., Rigonatti, S. P., Casoy, I., \& Barros, D. M. D. (2009). Perfil psicológico e comportamental de agressores sexuais de crianças. Revista de Psiquiatria Clínica, 36(3), 101-111. doi: 10.1590/S0101-60832009000300004

Shrout, P. E. \& Fleiss, J. L. (1979). Intraclass Correlations: Uses in Assessing Rater Reliability. Psychological Bulletin, 86(2), 420-428. doi: 10.1037//0033-2909.86.2.420

Soares, E. M. R., Silva, N. L., Matos, M. A. S., Araújo, E. T. H., Silva, L. R. \& Lago, E. C. (2016). Perfil da Violência Sexual Contra Crianças e Adolescentes. Revista Interdisciplinar, 9(1), 87-96. Recuperado de https://revistainterdisciplinar.uninovafapi.edu.br/index.php/revinter/ article/view/754

Stirpe, T. S., \& Stermac, L. E. (2003). An exploration of childhood victimization and family of origin characteristics of sexual offenders against children. International Journal of Offender Therapy and Comparative Criminology, 47(5), 542-555. doi: 10.1177/0306624X03253316

Waiselfisz, J. J. (2014). Mapa da violência 2014. Os jovens do Brasil. Rio de Janeiro (RJ): CEBELA-FLACSO.

Waiselfisz, J. J. (2015). Mapa da violência 2015. Mortes matadas por armas de fogo. Rio de Janeiro (RJ): CEBELA-FLACSO.

Ward, T., \& Beech, A. R. (2016). The Integrated Theory of Sexual Offending-Revised: A Multifield Perspective. Douglas. Em P. Boer. (Ed.) The Wiley handbook on the theories, assessment and treatment of sexual offending. Vol. I: Theories (pg. 123-137). John Wiley \& Son: New Jersey. doi: 10.1002/9781118574003.wattso006

Weiler, B. L., \& Widom, C. S. (1996). Psychopathy and violent behaviour in abused and neglected young adults. Criminal Behaviour and Mental Health, 6(3), 253-271. doi:10.1002/cbm.99

Widom, C. S., \& Ames, M. A. (1994). Criminal consequences of childhood sexual victimization. Child Abuse E Neglect, 18(4), 303-318. doi:10.1016/0145-2134(94)90033-7

Young, M. H., Justice, J. V., \& Edberg, P. (2010). Sexual offenders in prison psychiatric treatment: A biopsychosocial description. International Journal of Offender Therapy and Comparative Criminology, 54(1), 92-112. doi: 10.1177/0306624X08322373

\section{Sobre os autores}

Julia Nunes de Souza Teixeira é psicóloga (PUC Goiás), doutoranda do programa de pós-graduação em Psicologia Social (UERJ), Mestre em Psicologia (PUC Goiás), especialista em Psicologia Organizacional (UNIARA) e em Gestão Estratégica de Pessoas e Psicologia Organizacional pela Universidade Metodista de São Paulo.

Ana Cristina Resende é psicóloga (PUC Goiás) é doutora em Psicologia pela Universidade Federal do Rio Grande do Sul (UFRGS) e, atualmente, professora no Programa de Pós-Graduação Stricto Sensu em Psicologia da Pontifícia Universidade Católica de Goiás, Goiânia.

Rodrigo Perissinotto é psicólogo (PUC Goiás), doutorando e mestre em Psicologia pela PUC Goiás e, atualmente, é professor de graduação na Unidade de Biociências no Centro Universitário de Mineiros (UNIFIMES). 